\title{
Resource allocation for OFDM-based multiuser cooperative underlay cognitive systems
}

\author{
Marwa Chami* ${ }^{*}$, Mylene Pischella and Didier Le Ruyet
}

\begin{abstract}
This paper investigates the resource allocation problem for a multiuser underlay cognitive system where the secondary system is allowed to transmit and cooperate with the primary system. In this scenario, the secondary users are subject to two main constraints in the presence of the primary user: their total power budget and the allowable interference threshold at the primary receiver. Power and subcarrier allocation problems are detailed in order to maximize the system sum rate. In this work, we highlight the benefits of the proposed multiuser adaptive algorithm which encompasses three phases. The first step includes the adaptive selection of the decoding strategy at the secondary receiver which is either treating interference as noise or performing successive interference cancelation or superposition coding. The second step describes the subcarrier allocation among the different users. Finally, the third step details the optimal distribution of the available power budget on the users. The problem is first treated assuming perfect channel state information (CSI). The simulation results show that our proposed scheme achieves higher secondary and sum rates compared to existing approaches with perfect CSI. The robustness of the proposed algorithm when the secondary user has only partial information about channel gains is also derived.
\end{abstract}

Keywords: Cognitive radio, Underlay, Optimization, Maximum sum rate, OFDM, Multiuser, Imperfect CSI

\section{Introduction}

The idea of cognitive radios (CRs), which is a particular extension of software-defined radio that employs model-based reasoning about the channel, users, and communications context [1], was born out of the wireless spectrum's limitation. Advanced radio and signal processing technology along with novel spectrum allocation policies are used to support new wireless users operating in the existing spectrum without degrading the performance of licensed users called primary users (PUs) [2]. More specifically, the CR technology permits an unlicensed user called a secondary user (SU) to coexist with the PU without degrading his performance. In a CR system, the SUs have the ability to sense and adapt to their environment in order to detect possible frequency holes in the wireless spectrum which therefore increase the spectral efficiency. Consequently, the CR technology is very appealing since

${ }^{*}$ Correspondence: marwa@elchami.com

CEDRIC/LAETITIA Laboratory, Conservatoire National des Arts et Métiers

(CNAM), 292 Rue Saint-Martin 75003 Paris, France it provides a low-cost and highly flexible alternative to the classic single-frequency band and single-protocol wireless communication [3].

There exist three types of CR systems: interweave, underlay, and overlay cognitive radio. In an interweave scenario, the SUs adaptively utilize the spectrum band whenever it is not used by the PU [4]. In the underlay case, the SUs are allowed to coexist with the PU provided that the interference caused to the PU is below a predefined threshold [4]. In the latter category, the secondary system has the knowledge of the primary system codebook, channel gains, and transmitted information. A part of the SUs' power can be used to retransmit the PU message so that the SUs are able to maintain or improve the primary rate while achieving their own communication goals [4]. Both overlay and underlay scenarios are compared in [5].

Multi-carrier transmissions such as orthogonal frequency-division multiplexing (OFDM) are largely used for CR networks due to their flexibility in allocating resources among SUs [6]. Adaptive resource allocation for the OFDM systems has been studied extensively 
during the past two decades. A comprehensive survey can be found in [7] and references therein. Moreover, resource allocation for OFDM-based CR networks has attracted much attention recently. An overview of the state-of-the-art research results can be found in [8]. This issue has been studied for both single-user and multiuser cases. For the single-user case, optimal and suboptimal power allocation schemes were proposed to maximize the sum capacity of the CR system under the interference constraints of the PUs in [9]. In [10], a low-complexity optimal power allocation algorithm was derived by exploiting the structure of the considered optimization problem. A greedy max-min algorithm was proposed to maximize the throughput of the CR system with a given power budget in [11]. In [12], the authors aimed to maximize the CR network throughput under interference limit and total power budget constraints. The optimal power allocation to achieve ergodic capacity and outage capacity in fading channel was derived in [13]. In [14], the authors maximized the secondary achievable rate under a new criterion referred to as rate-loss constraint. The authors in [15] investigated the problem of random subcarrier allocation in OFDM-based CR networks without any spectrum-sensing information at the SU. The SU's transmit power was adjusted in order to maintain the interference on the primary receiver under a predefined threshold. The downlink resource allocation problem in a spectrum-sharing environment is studied in [16]. A time averaging is introduced to approximate the interference constraints for both short and long terms. In [17], the authors have characterized a capacity region of a CR system assuming that the secondary transmitter has side information about the primary transmission. They supposed that the SU has to satisfy two conditions in order to be able to coexist with the PU: (i) no interference is accepted at the primary system and (ii) the primary receiver is unaware of the presence of the cognitive system.

In [18], a heuristic scheme is proposed where the secondary receiver either treats the interference as noise or uses successive interference cancelation (SIC) or is switched off. The achievable rate is studied under the constraint that (i) an interference level is allowable on the PU and (ii) the primary encoder-decoder pair is unaware of the presence of cognitive radio. We have extended this work in [19] by proposing a new algorithm where the cognitive user can also use superposition coding (SC) [20] under certain conditions. The same problem was investigated in [21] by optimally allocating the SU power using gradient descent algorithm.

Resource allocation for a multiuser OFDM-based CR system was investigated in [22-27]. The authors in [22] made use of subcarrier allocation to improve the performance of cognitive networks by exploiting the so-called multiuser interference diversity. They considered that the SU with the largest achievable receiver signal to noise ratio is selected among all the SUs. In [23], a low-complexity algorithm is developed to maximize the sum capacity of a CR system with proportional rate constraints. The proposed algorithm exhibits a trade-off between capacity and fairness by jointly considering channel gain and the interference to the PUs. The multiuser diversity in an interweave scenario was studied in [24]. In [25], two fast resource allocation algorithms were derived for both real-time and non-real-time services in multiuser OFDMbased CR networks. In [26], the sum capacity of a multiuser OFDM-based CR system was maximized while satisfying the SUs' proportional rate requirements. The adaptive resource allocation problem in multiuser OFDMbased CR networks with imperfect spectrum sensing was investigated in [27]. The problem was addressed in two steps: subcarrier allocation and then power allocation for subcarriers.

Most of the previous cited works considered perfect CSI at the secondary transmitter. However, obtaining perfect knowledge about the channel gains at the secondary user is very difficult to implement in practice. The impact of imperfect CSI was investigated in [28-30] with uncertainty, quantization, or estimation errors. In [31], Suraweera et al. investigated the impact of imperfect CSI of the SU-PU link on the SU mean capacity, considering the effect of CSI quantization with a finite number of quantization levels. The ergodic capacity maximization problem with quantized information about CSI available at the SU through a limited feedback link was studied in [28], while optimum power strategy and ergodic capacity were derived under outage constraints in [29]. The impact of noisy CSI on spectral efficiency of multiuser multi-carrier CRs was considered in [30], where novel interference management schemes were derived based on different average-case and worst-case models of channel estimation error.

In this paper, we use the Han-Kobayashi strategy [32] for an underlay cooperative CR system composed of one secondary cell with many SUs. We propose a resource allocation algorithm for the multiuser system, in which the decoding strategies at the secondary receivers are defined and the power allocation is optimized in order to improve the sum rate of the system with perfect CSI. Without loss of generality, we carry out a statistical study for the single-user case with perfect CSI. We further prove via numerical/simulation evaluation the robustness of the proposed algorithm when imperfect CSI on both primary links is considered at the secondary user. The main contributions of the paper are highlighted below:

- A heuristic approach is proposed for a multiuser underlay cognitive radio system. This heuristic is 
designed for the perfect CSI scenario by considering a cooperative underlay cognitive system. A complete resource allocation problem is addressed in three steps:

- The decoding strategies are identified per subcarrier for each user according to several conditions that will be defined later.

- A subcarrier allocation algorithm is described to choose the active subcarriers per user. Only one $\mathrm{SU}$ is activated per subcarrier following a criterion derived from the single-user algorithm.

- An optimization problem is then formulated, independently for each user, in order to maximize the secondary rate and the sum rate of the system under the constraint of power budget of each user and the maximum allowable interference on the PU.

- Motivated by the alternating optimization method [33], we propose an approach that sequentially solves a feasibility problem using dual decomposition.

- We identify several decoding strategy areas for different scenarios based on the mobile positions when two users exist in the secondary cell.

- We investigate the same problem for two secondary users under the assumption that imperfect CSI on the links between both primary and secondary transmitters and the primary receiver is available at the secondary user. This study can be easily extended to more than two secondary users.

The rest of this paper is organized as follows. Section 2 describes the system model and gives an overview on the decoding strategies at the receivers. Section 3 explains the proposed algorithm to solve the resource allocation problem with perfect CSI. Section 4 investigates the problem when imperfect CSI is available at the SU. Section 5 evaluates the performance of the proposed algorithm with both perfect and imperfect CSI. Finally, Section 6 concludes the paper.

\section{System model and problem formulation}

\subsection{System model}

We consider a cognitive radio system model composed of one primary cell and one secondary cell which may contain several SUs. This system model can be seen as a cooperative underlay cognitive radio, due to the cooperation between the systems and the allowed interference threshold on the primary. The primary system occupies a licensed bandwidth $B$ which is divided into $L$ adjacent and parallel subcarriers. The secondary base station (BS) is located at a distance $d_{\text {sec }}$ from the primary BS. In the considered scenario, the SUs need to adapt their decoding strategies to avoid disturbing the PU. Consequently, they have to transmit either when the primary system is off or under an interference constraint fixed by the PU. Thus, the secondary transmitters use channel interweave when the subbands are currently left vacant by the primary system; otherwise, they use channel underlay. Unless stated otherwise, perfect CSI knowledge is considered throughout this work at each node. We also assume perfect sensing of primary activity. The cooperation between the primary and secondary systems can be provided by a band manager [34]. We study the uplink transmission in a given time slot. In the sequel, index $p$ refers to the primary system, while index $s$ refers to the secondary BS. $s_{u_{[k]}}$ denotes the secondary transmitter that transmits on the $k$-th subcarrier. The received primary and secondary signals in each subcarrier $k \in\{1, \ldots, L\}$ can be written as (see Fig. 1)

$$
\begin{aligned}
& y_{p}^{k}=h_{p p}^{k} x_{p}^{k}+h_{p s_{u_{[k]}}}^{k} x_{s_{u_{[k]}}}^{k}+z_{p}^{k} \\
& y_{s}^{k}=h_{s p}^{k} x_{p}^{k}+h_{s s_{u_{[k]}}}^{k} x_{s_{u_{[k]}}}^{k}+z_{s}^{k}
\end{aligned}
$$

where $y_{i}^{k}$ is the channel output and $x_{i}^{k}$ is the channel input corresponding to data $s_{i}^{k}$ with power $P_{i}^{k}$ for subcarrier $k$ and user $i$. $P_{i, \max }$ is the maximum transmit power of transmitter $i$. $h_{i j}^{k}$, a zero-mean complex circular Gaussian variable, denotes the channel gain between transmitter $j$ and receiver $i$. The channel gains are assumed to be constant during a transmission time slot. $z_{i}^{k}$ denotes the additive white Gaussian noise at receiver $i$. The noise variance $n_{i}^{k}=n_{0}$ is the same on each subcarrier $k$.

\subsection{Problem formulation}

In this work, we will consider the case of a single PU in the primary cell. We further assume that the primary receiver always treats the interference from the SUs as noise. Our goal is to maximize both primary and secondary achievable rates. The primary achievable rate is maximized

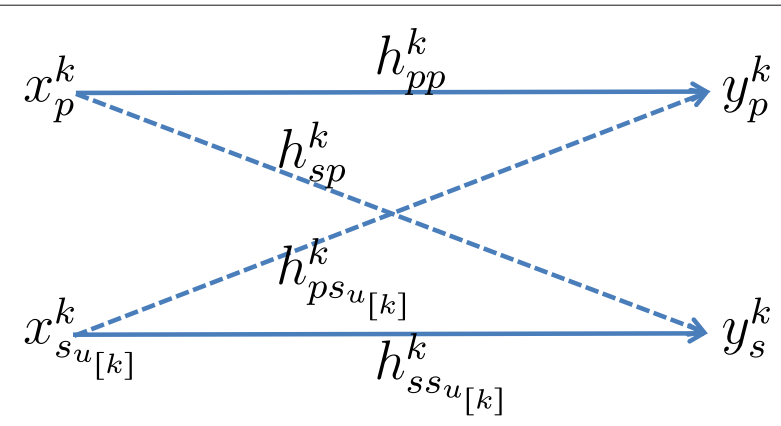

Fig. 1 Underlay cognitive channels on subcarrier $k \in\{1, \ldots, L\}$ 
under total power constraint. The optimization problem for the PU can be formulated as

$$
\begin{aligned}
& \max _{\mathbf{P}_{p}} \sum_{k=1}^{L} \frac{B}{L} \log _{2}\left(1+\frac{\left|h_{p p}^{k}\right|^{2} P_{p}^{k}}{n_{0}+\left|h_{p s_{u_{[k]}}}^{k}\right|^{2} P_{s_{u_{[k]}}}^{k}}\right) \\
& \text { s.t. } \sum_{k=1}^{L} P_{p}^{k} \leq P_{p, \max }
\end{aligned}
$$

where $\mathbf{P}_{p}$ denotes the power vector for user $p$. Problem (1) has been greatly investigated in the literature, and the optimal solution is given by the waterfilling solution [35]. For the SU, the per subcarrier interference must remain below a predefined threshold $I_{\text {th }}^{k}$. Under this condition, we address the resource allocation problem in order to maximize the secondary cell achievable rate. Defined $\mathbf{P}_{s_{u_{[k]}}}$ as the power vector for user $s_{u_{[k]}}$, the secondary resource allocation problem is formulated as

$$
\begin{aligned}
& \max _{\mathbf{P}_{s_{\left.u_{[k]}\right]}}} \sum_{k=1}^{L} \sum_{u=1}^{U} R_{s_{u_{[k]}}}\left(P_{p}, P_{s}, \theta_{u}^{k}\right) \\
& \text { s.t. } \sum_{k=1}^{L} P_{s_{u_{[k]}}}^{k} \leq P_{s, \max }, \forall u \\
& \text { s.t. } \sum_{u=1}^{U} \theta_{u}^{k} \leq 1, \forall k \\
& \text { s.t. } \theta_{u}^{k} \in\{0,1\}, \forall k, \forall u
\end{aligned}
$$

where $R_{s_{u_{[k]}}}$ denotes the SU achievable rate and $\theta_{u}^{k}$ the subcarrier assignment parameter. Its explicit expression depends on the decoding strategies used by the SUs, and it will be given in the subsequent sections.

\section{Resource allocation with perfect CSI \\ 3.1 Description of the algorithm}

Our proposed algorithm is executed alternatingly between the primary and the secondary systems. More specifically, given a starting power allocation $\mathbf{P}_{p, 0}$ and $\mathbf{P}_{s, 0}$, the SUs apply a per subcarrier decoding strategy which depends on the primary and SUs' power allocation obtained from the iteration $n-1$. Based on the applied decoding strategy, an achievable rate is computed for each $\mathrm{SU}$ on each subcarrier. Finally, the SU with the highest rate is activated within subcarrier $k$, and the SU power optimization problem is solved.

A summary of our proposed scheme is given in Algorithm 1, where at the $n$-th iteration, $\mathbf{P}_{i, n}=$ $\left(P_{i, n}^{1}, \ldots, P_{i, n}^{L}\right)^{T}$, with $i=\left\{p, s_{u_{[k]}}\right\}$ and $\mathbf{P}_{n} \in$ $\left\{\mathbf{P}_{p, n}, \mathbf{P}_{s_{u_{[k]}}, n}\right\}$.

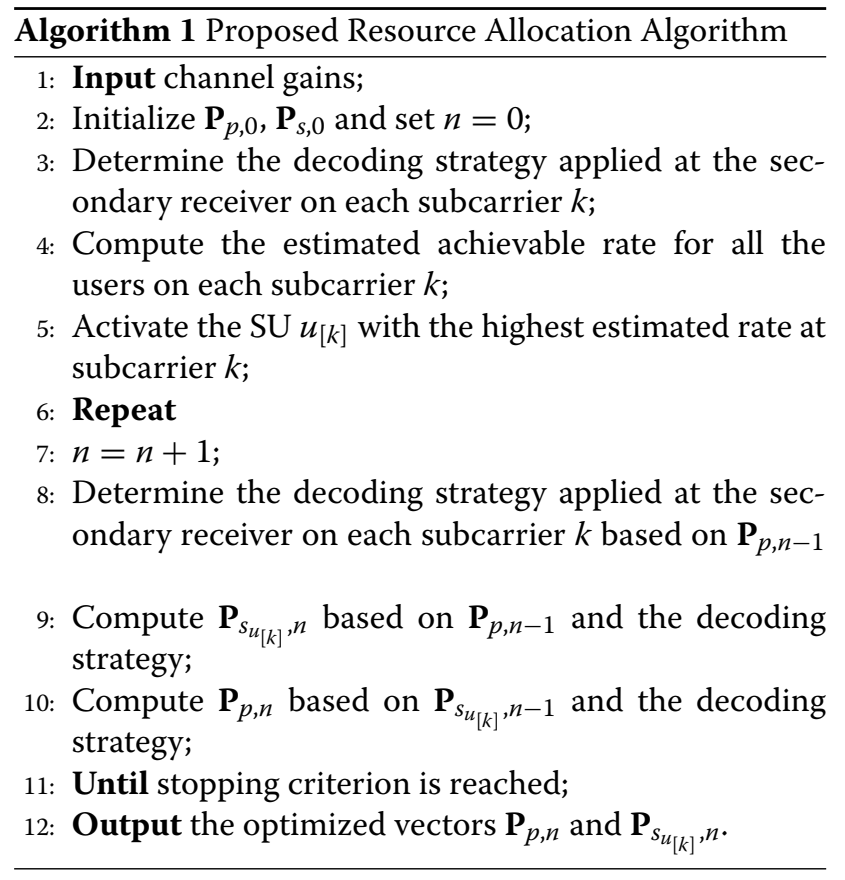

\subsection{Decoding strategies and achievable rates at the secondary receiver}

If $P_{p,(n-1)}^{k} \neq 0$, different decoding strategies for each SU are identified based on the interference level at each SU. The decoding strategy on each subcarrier $k$ at the $n$-th iteration is defined according to the PU's power and each SU's power in the previous iteration. These power levels are optimized using waterfilling and Lagrangian methods for the PU and SUs, respectively, as will be detailed in the next section. On each SU, different decoding strategies can be applied under certain conditions. The different possible decoding strategies are:

1. Strategy 1: If $P_{p,(n-1)}^{k}=0$, the secondary receiver decodes its message error-free and the secondary rate is defined by

$$
R_{s_{[k]}, n}^{k}=\frac{B}{L} \log _{2}\left(1+\frac{\left|h_{s s_{u_{[k]}}}^{k}\right|{ }^{2} P_{s_{\left.u_{[k]}\right]}, n}^{k}}{n_{0}}\right)
$$

In the rest of the paper, this strategy is called "interweave."

2. Strategy 2: If $P_{p,(n-1)}^{k} \neq 0$ and $\left|h_{s p}^{k}\right|^{2}<\left|h_{s s_{\left.u_{[k]}\right]}}^{k}\right|^{2}$, the interference to the $\mathrm{SU}$ is weak and is treated as noise. The secondary rate is given by

$$
R_{s_{\left.u_{[k]}\right]}, n}^{k}=\frac{B}{L} \log _{2}\left(1+\frac{\left|h_{s s_{u_{[k]}}}^{k}\right|^{2} P_{s_{\left.u_{[k]}\right]}, n}^{k}}{\left|h_{s p}^{k}\right|^{2} P_{p, n-1}^{k}+n_{0}}\right)
$$


3. Strategy 3: If $P_{p,(n-1)}^{k} \neq 0$ and $\left|h_{s p}^{k}\right|^{2} \geqslant\left|h_{s s_{\left.u_{[k]}\right]}^{k}}^{k}\right|^{2}$ the interference on the $\mathrm{SU}$ is strong. Therefore, if

$$
a^{k} P_{s_{u_{[k]}}}^{k} \geq c^{k}
$$

is verified, SIC can be applied [36], where

$$
\begin{aligned}
a^{k} & =\left|h_{s p}^{k}\right|^{2}\left|h_{p s_{\left.u_{[k]}\right]}}^{k}\right|^{2}-\left|h_{p p}^{k}\right|^{2}\left|h_{s s_{\left.u_{[k]}\right]}}^{k}\right|^{2} \\
c^{k} & =n_{0}\left(\left|h_{p p}^{k}\right|^{2}-\left|h_{s p}^{k}\right|^{2}\right)
\end{aligned}
$$

In this case, the achievable secondary rate is

$$
R_{s_{u_{[k]},}, n}^{k}=\frac{B}{L} \log _{2}\left(1+\frac{\left|h_{s s_{\left.u_{[k]}\right]}}^{k}\right|^{2} P_{s_{\left.u_{[k]}\right]}, n}^{k}}{n_{0}}\right)
$$

4. Strategy 4: If $P_{p,(n-1)}^{k} \neq 0$ and $\left|h_{s p}^{k}\right|^{2} \geqslant\left|h_{s s_{u_{[k]}}}^{k}\right|^{2}$ but (5) is not verified, the ability to apply $\mathrm{SC}$ at the $\mathrm{SU}$ is tested subject to the validation of the following set of inequalities [19]

$$
\begin{aligned}
& \frac{\left(\left|h_{p p}^{k}\right|^{2}-\left|h_{s p}^{k}\right|^{2}\right)}{\left|h_{p s_{u_{[k]}}}^{k}\right|^{2}\left|h_{s p}^{k}\right|^{2}}<\frac{P_{s_{u_{[k]}}}^{k}}{n_{0}} \\
& P_{s_{u_{[k]}}^{k} a^{k}<c^{k}}^{k}
\end{aligned}
$$

Thus, the secondary achievable rate can be given by [19]

$$
\begin{aligned}
R_{s_{\left.u_{[k]}\right]}, n}^{k} & =\frac{B}{L} \log _{2}\left(1+\frac{\alpha^{k}\left|h_{s s_{u_{[k]}}}^{k}\right|^{2} P_{s_{\left.u_{[k]}\right]}, n}^{k}}{n_{0}}\right) \\
& +\frac{B}{L} \log _{2}\left(1+\frac{\left(1-\alpha^{k}\right)\left|h_{\left.s u_{[k]}\right]}^{k}\right|^{2} P_{s_{\left.u_{[k]}\right]}^{k}, n}^{k}}{\alpha^{k}\left|h_{s s_{[k]}}^{k}\right|{ }^{2} P_{s_{\left.u_{[k]}\right]}}^{k}+\left|h_{s p}^{k}\right|^{2} P_{p, n}^{k}+n_{0}}\right)
\end{aligned}
$$

with

$$
\alpha^{k}=\frac{\left(\left|h_{s p}^{k}\right|^{2}-\left|h_{p p}^{k}\right|^{2}\right) n_{0}+\left|h_{p s_{u_{[k]}}}^{k}\right|{ }^{2}\left|h_{s p}^{k}\right|^{2} P_{s_{\left.u_{[k]}\right]}, n}^{k}}{\left|h_{p p}^{k}\right|^{2}\left|h_{s s_{u_{[k]}}}^{k}\right|^{2} P_{s_{u_{[k]}}, n}^{k}}
$$

Eqs. (7a) to (9) are detailed in Appendices 1, 2, and 3.

5. Strategy 5: If $P_{p,(n-1)}^{k} \neq 0$ and $\left|h_{s p}^{k}\right|^{2} \geqslant\left|h_{s s_{[k]}}^{k}\right|^{2}$ but neither SIC nor SC can be applied, the SU is turned off.

These different decoding strategies lead to four different expressions for the secondary rate defined in different

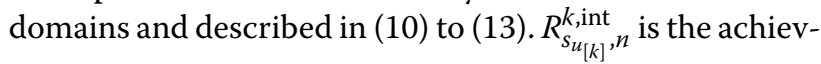
able rate when the interference is treated as noise. $R_{s_{u_{[k]}}, n}^{k, \text { SIC }}$ represents both SIC and interweave cases and $R_{s_{u_{[k]}, n}}^{k, \mathrm{SC}}$ the achievable rate when SC is applied. $R_{s_{\left.u_{[k]}\right]}, n}^{k, f f}$ denotes the null rate when the secondary transmitter is turned off.

$$
\begin{aligned}
& R_{s_{\left.u_{[k]}\right]}, n}^{k \text { int }}=\frac{B}{L} \log _{2}\left(1+\frac{\left|h_{s s_{[k]}}^{k}\right|^{2} P_{s_{u_{[k]}}, n}^{k}}{\left|h_{s p}^{k}\right|^{2} P_{p, n-1}^{k}+n_{0}}\right), k \in \mathcal{S}_{2} \\
& R_{s_{\left.u_{[k]}\right]}, n}^{k, \mathrm{SIC}}=\frac{B}{L} \log _{2}\left(1+\frac{\left|h_{s s_{\left.u_{[k]}\right]}}^{k}\right|^{2} P_{s_{\left.u_{[k]}\right]}, n}^{k}}{n_{0}}\right), k \in \mathcal{S}_{1} \text { and } \mathcal{S}_{3} \\
& R_{s_{u_{[k]}, n}}^{k, \mathrm{SC}}=R_{s_{\left.u_{[k]}\right]}, n, 1}^{k, \mathrm{SC}}+R_{s_{\left.u_{[k]}\right]}^{k, \mathrm{SC}}}^{k, 2}, k \in \mathcal{S}_{4} \\
& R_{s_{u_{[k]}}, n}^{k, \text { off }}=0, k \in \mathcal{S}_{5}
\end{aligned}
$$

where

$R_{s_{\left.u_{[k]}\right]}^{k, S C}, 1}^{k}=\frac{B}{L} \log _{2}\left(1+\frac{\frac{\left|h_{p s u_{[k]}}^{k}\right|^{2}\left|h_{s p}^{k}\right|^{2}}{\left|h_{p p}^{k}\right|^{2}} P_{s_{\left.u_{[k]}\right]}^{k}}^{k}+\frac{\left(\left|h_{s p}^{k}\right|^{2}-\left|h_{p p}^{k}\right|^{2} n_{0}\right.}{\left|h_{p p}^{k}\right|^{2}}}{n_{0}}\right)$

and

$R_{s_{u_{[k]},}, \mathrm{SC}, 2}^{k}$

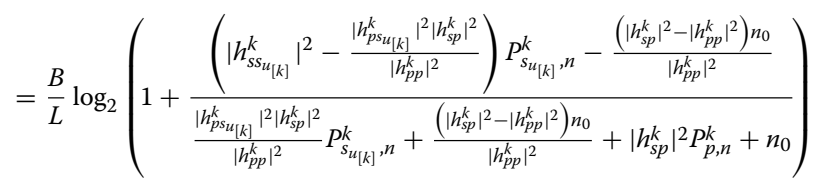

( $\mathcal{S}_{1}=\{k \in\{1, \ldots, L\}$ for "Interweave" $\}$

$\mathcal{S}_{2}=\{k \in\{1, \ldots, L\}$ when interference is treated as a noise $\}$

$\left\{\mathcal{S}_{3}=\{k \in\{1, \ldots, L\}\right.$ for "SIC" $\}$

$\mathcal{S}_{4}=\{k \in\{1, \ldots, L\}$ for "SC" $\}$

$\mathcal{S}_{5}=\{k \in\{1, \ldots, L\}$ when the secondary is turned off $\}$

\subsection{Subcarrier allocation}

We start by assuming perfect PU-PU and SU-PU channel link knowledge available at the SU. We evaluate within each subcarrier the data rate that can be achieved in all the links between SUs and the secondary BS by assuming equal power allocation. The data rate $\hat{R}_{u_{[k]}}^{k}$ is obtained depending on the decoding strategy applied in the subcarrier and by taking into account the power limitation due to the interference threshold. This rate is defined in Eqs. (10) to (13), where equal power allocation is also set in each subcarrier of the PU. For each subcarrier, the SU with the highest estimated rate is activated.

\subsection{Power allocation optimization}

The power allocation procedure is summarized in Fig. 2. At the $n$-th iteration, a test is done in order to define the decoding strategy used at the secondary receiver. The achievable rate per subcarrier is calculated according to aforementioned cases and is used to select one of the SUs within subcarrier $k$ and then to optimize the power allocation for the active SU. Once $R_{s_{\left.u_{[k}\right]}, n}^{k}$ is determined for all subcarriers, the PU can update its power $\mathbf{P}_{p, n}$ using waterfilling and based on the measured interference from the previous iteration, $\mathbf{P}_{s_{\left.u_{[k]}\right]}, n-1}$. Similarly, the secondary system updates its power $\mathbf{P}_{s_{\left.u_{k k}\right]}}, n$ using dual decomposition 


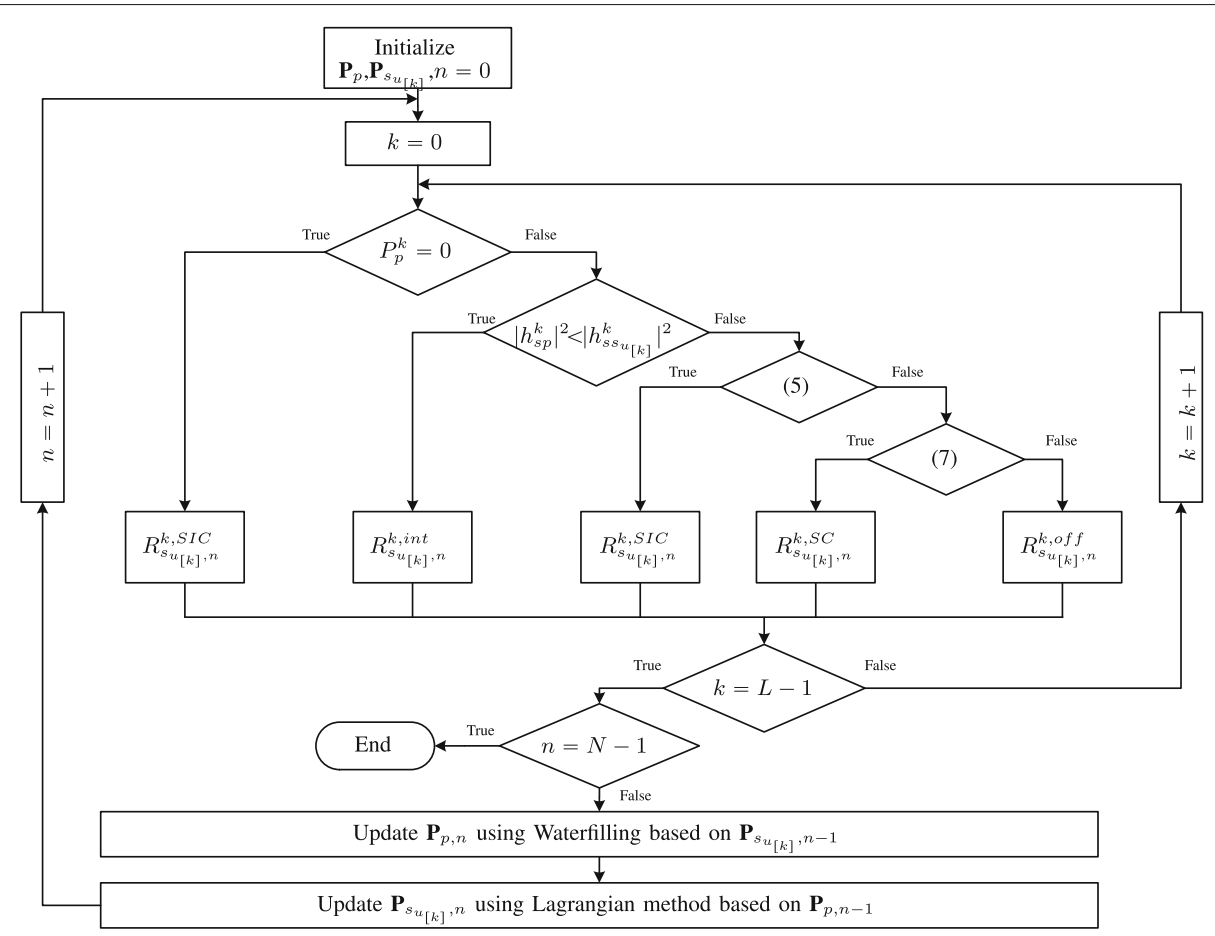

Fig. 2 Power allocation procedure

process, based on $\mathbf{P}_{p, n-1}$. The stopping criterion for Algorithm 1 is either $\frac{\left|R_{s_{[k]}, n}-R_{s_{\left.u_{[k]}\right]}, n-1}\right|}{\left|R_{s_{\left.u_{k k}\right]}, n-1}\right|}<10^{-4}$ or $n=10$ iterations. This alternative process is repeated $N$ times. Note that the convergence of the power optimization algorithm is guaranteed even if the primary power control is not synchronized with the secondary power control, as proven in [37].

We assume that optimization is performed at the BS (primary and secondary, respectively). Regarding secondary transmission, the decoding strategy and the power allocation is performed by the secondary BS based on the channel gains and on the transmit power of the previous iteration $(n-1)$. They are then transmitted to the secondary user on a feedback channel (identifying which decoding strategy and which power level), so that the secondary user can use them at the next Transmission Time Interval (TTI) that includes several time slots. In order to implement this, we shall assume that the channel coherence time is large and that the fading values do not vary over at least two consecutive uplink-downlink TTI. This assumption is valid for low to medium speeds of the mobile users.

Now, we investigate the power allocation problem for the SU. The objective is to optimally choose the power vector $\mathbf{P}_{s_{\left.u_{[k]}\right]}}$ in order to maximize the secondary rate, while satisfying the interference and the power constraints. If the interference constraints are not met, the subcarrier is switched off. The power control problem is solved iteratively. The decoding strategy and power allocation are run for each SU independently.

The optimization problem for each user $u_{[k]}$ at the $n$-th iteration can be expressed by:

$$
\begin{aligned}
& \max _{\left\{\mathbf{P}_{\left.u_{[k]}, n\right\}}\right.} R_{s}, n \\
& \text { s.t. } \sum_{k \in \mathcal{S}_{u_{[k]}}} P_{s_{\left.u_{[k]}\right]}, n}^{k} \leqslant P_{s_{u_{[k]}}, \max } \\
& \text { s.t. } P_{s_{\left.u_{[k]}\right]}, n}^{k} \geqslant 0, \forall k \in \mathcal{S}_{u_{[k]}} \\
& \text { s.t. }\left|h_{p s_{u_{[k]}}}^{k}\right|^{2} P_{s_{u_{[k]}}, n}^{k} \leqslant I_{\text {th }}^{k}, \forall k \notin \mathcal{S}_{1} \\
& \text { s.t.(5), } \forall k \in \mathcal{S}_{u_{[k]}} \text { in SIC case } \\
& \text { s.t.(7), } \forall k \in \mathcal{S}_{u_{[k]}} \text { in SC case }
\end{aligned}
$$

where $R_{s, n}=\sum_{k \in \mathcal{S}_{2}} R_{s, n}^{k, \text { int }}+\sum_{k \in \mathcal{S}_{1} \cap \mathcal{S}_{3}} R_{s, n}^{k, \text { SIC }}+$ $\sum_{k \in \mathcal{S}_{4}} R_{s, n}^{k, S C}$ and $\mathcal{S}_{u_{[k]}}$ is the set of subcarriers allocated to user $u_{[k]}$.

Since the optimization problem is separable, it can be efficiently solved using the Lagrange dual decomposition method by decomposing the original problem into three subproblems, depending on the decoding strategies applied at the SU.

The Lagrangian of problem (14) can be written as

$\mathcal{L}\left(P_{s, n}^{k}, \mu_{s, n}\right)=-R_{s, n}+\mu_{s, n}\left(\sum_{k=1}^{L} P_{s, n}^{k}-P_{s, \max }\right)$ 
with $\mu_{s, n} \geq 0$ being a Lagrange multiplier. Let $D$ be the set specified by the remaining constraints in (14d), (14e), and (14f). Consequently, problem (14) can be given by the subproblems $C_{\mathrm{int}}, C_{\mathrm{SIC}}$, and $C_{\mathrm{SC}}$ defined over $\mathcal{S}_{2}, \mathcal{S}_{3}$, and $\mathcal{S}_{4}$, respectively, as

$$
\begin{aligned}
& C_{\text {int }}: \max _{\left\{P_{s_{\left.u_{[k}\right]}^{k}, n}^{k}\right\}_{k \in \mathcal{S}_{2}}} \sum_{k \in \mathcal{S}_{2}} R_{s_{\left.u_{[k]}\right]}, n}^{k \text { int }}-\mu_{s, n} \sum_{k \in \mathcal{S}_{2}} P_{s_{\left.u_{[k]}\right]}, n}^{k} \\
& \left|h_{p s_{\left.u_{[k]}\right]}}^{k}\right|^{2} P_{s_{u_{[k]},}, n}^{k} \leqslant I_{\mathrm{th}}^{k}, \forall k \in \mathcal{S}_{2} \\
& C_{\mathrm{SIC}}: \max _{\left\{P_{s_{\left.u_{[k}\right]}, n}^{k}\right\}_{k \in \mathcal{S}_{3}}} \sum_{k \in \mathcal{S}_{3}} R_{s_{\left.u_{[k]}\right]}, n}^{k, \mathrm{SIC}}-\mu_{s, n} \sum_{k \in \mathcal{S}_{3}} P_{s_{\left.u_{[k}\right]}, n}^{k} \\
& \left|h_{p s_{u_{[k]}}}^{k}\right|^{2} P_{s_{\left.u_{[k}\right]}, n}^{k} \leqslant I_{\mathrm{th}}^{k}, \forall k \in \mathcal{S}_{3} \\
& a^{k} P_{s_{u_{[k]}}^{k}}^{k} \geq c^{k}, \forall k \in \mathcal{S}_{3} \\
& C_{\mathrm{SC}}: \max _{\left\{P_{\left.s_{[k]}\right]}^{k}, n\right\}_{k \in \mathcal{S}_{4}}} \sum_{k \in \mathcal{S}_{4}} R_{s_{\left.u_{[k]}\right]}, n}^{k, \mathrm{SC}}-\mu_{s, n} \sum_{k \in \mathcal{S}_{4}} P_{s_{\left.u_{[k]}\right]}, n}^{k} \\
& \left|h_{p s_{u_{[k]}}}^{k}\right|^{2} P_{s_{\left.u_{[k]}\right]}, n}^{k} \leqslant I_{\text {th }}^{k}, \forall k \in \mathcal{S}_{4}
\end{aligned}
$$

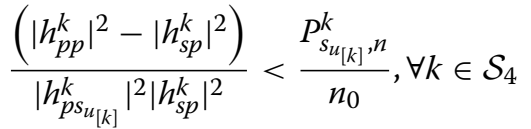

$$
\begin{aligned}
& a^{k} P_{s_{u_{[k]}}}^{k}<c^{k} \forall k \in \mathcal{S}_{4}
\end{aligned}
$$

where the subtracted term in the objective function represents the total power constraint which is common for all subproblems. It can be verified that subproblem $C_{\text {int }}$ and subproblem $C_{\mathrm{SIC}}$ are convex problems. On the other hand, $R_{s_{\left.u_{[k]}\right]}, n, 2}^{k, \mathrm{SC}}$ is not concave in $P_{s_{\left.u_{[k]}\right]}, n}^{k}$. Thus, $R_{s_{\left.u_{[k]}\right]}^{k, ~}, n}^{k}$ is not concave and the subproblem $C_{\mathrm{SC}}$ is not a convex problem. $R_{s_{u_{[k]}}, n, 2}^{k, \mathrm{SC}}$ can then be approximated using the first order Taylor approximation at a feasible point $\overline{P_{s_{u_{[k]},},}^{k}}$, in order to have a convex optimization problem. Once we obtain a convex problem for $R_{s, n}$ and since the optimization problem of $R_{p, n}$ is convex, the global problem can be handled by alternating optimization method [38].

The approximated function of this term can be given by

$$
\begin{aligned}
R_{s_{\left.u_{[k]}\right]}, n, 2}^{k, \mathrm{SC}} \simeq & \left.R_{s_{\left.u_{[k]}\right]}^{k, \mathrm{SC}}, n, 2}^{k, \overline{P_{s_{u_{[k]}}, n}^{k}}}\right)+B_{n}^{k}\left(\overline{P_{s_{u_{[k]}}, n}^{k}}\right) \\
& \times\left(P_{s_{u_{[k]}}^{k}, n}^{k}-\overline{P_{s_{u_{[k]}}, n}^{k}}\right)
\end{aligned}
$$

with

$$
\begin{aligned}
& B_{n}^{k}=\frac{\partial R_{s_{[k]}, n, 2}^{k, \mathrm{SC}}}{\partial P_{s_{\left.u_{[k]}\right]}}^{k}, n}
\end{aligned}
$$

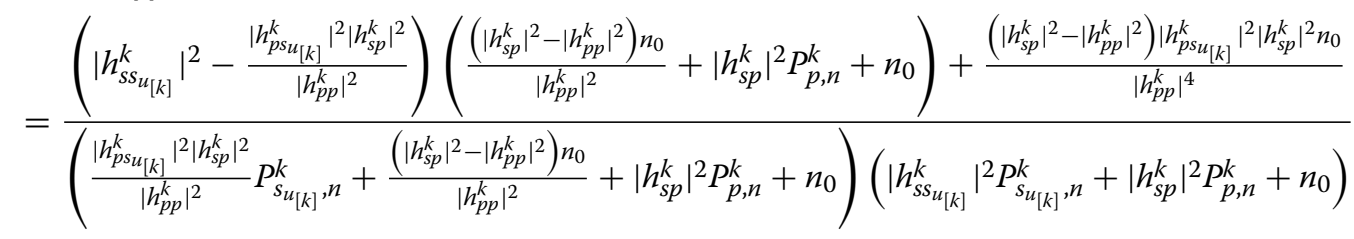

Besides, we demonstrate that the objective function is strictly increasing in $\mathbf{P}_{s, n}$. Then, the problem can be transformed into an equality-constrained problem, and the stopping condition becomes

$$
\sum_{k=1}^{L} P_{s, n}^{k}=P_{s, \max }
$$

Thus, an analytical solution of all subproblems can be obtained with the Karush-Kuhn-Tucker conditions [39].

To solve subproblem $C_{\mathrm{SC}}$, let define the unconstrained subproblem

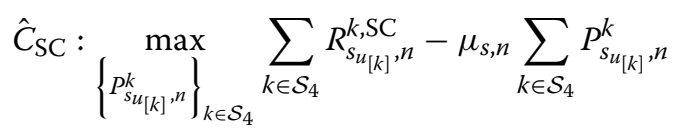

with Lagrangian

$$
\hat{\mathcal{L}}\left(P_{s, n}^{k}, \mu_{s, n}\right)=-\sum_{k \in \mathcal{S}_{4}} R_{s_{\left.u_{[k]}\right]}, n, \mathrm{SC}}^{k}+\mu_{s, n} \sum_{k \in \mathcal{S}_{4}} P_{s_{[[k]}, n}^{k}
$$

Let $\hat{f}$ be the partial derivative of $\hat{\mathcal{L}}$ with respect to $P_{s, n}^{k}$.

$$
\begin{aligned}
\hat{f}\left(P_{s, n}^{k}, \mu_{s, n}\right)= & -\frac{\left|h_{p s_{u_{[k]}}}^{k}\right|^{2}}{\left|h_{p s_{u_{[k]}}}^{k}\right|{ }^{2} P_{s_{u_{[k]}}, n}^{k}+n_{0}}-B^{k}\left(\overline{P_{s_{\left.u_{k}\right]}, n}^{k}}\right) \\
& +\mu_{s, n}=0
\end{aligned}
$$

Thus, the solution of subproblem $\hat{C}_{\mathrm{SC}}$ can be given by

$$
\hat{P}_{s_{[k]}, n}^{k}=\left[\frac{1}{\mu_{s, n}-B^{k}\left(\overline{P_{s_{\left.u_{[k]}\right]}, n}^{k}}\right)}-\frac{n_{0}}{\left|h_{p s_{u_{[k]}}}^{k}\right|^{2}}\right]^{+}
$$

which can be expressed by

$$
\hat{P}_{s_{u_{[k]}, n}}^{k}=\left[\frac{1}{\hat{\mu}_{s, n}}-\frac{n_{0}}{\left|h_{p s_{u_{[k]}}}^{k}\right|^{2}}\right]^{+}
$$

where

$$
\hat{\mu}_{s, n}=\frac{L}{B}\left(\mu_{s, n}-B^{k}\left(\overline{P_{s_{u_{[k]}}, n}^{k}}\right)\right)
$$


Taking into consideration the constraints of subproblem $C_{\mathrm{SC}}$, its general solution $P_{s_{\left.u_{[k]}\right]}^{k, n C}}^{,, n a s}$ to be

$$
n_{0} \frac{\left(\left|h_{p p}^{k}\right|^{2}-\left|h_{s p}^{k}\right|^{2}\right)}{\left|h_{p s_{\left.u_{[k]}\right]}}^{k}\right|^{2}\left|h_{s p}^{k}\right|^{2}}<P_{s_{u_{[k]}}, n}^{k}<\min \left\{\frac{c^{k}}{a^{k}}, \frac{I_{\text {th }}^{k}}{\left|h_{p s_{u_{[k]}}}^{k}\right|^{2}}\right\}
$$

which depends on the sign of $a^{k}$ and $c^{k}$. (See also Table 1 in [19].)

The solution of problem (16) is directly provided by waterfilling for both subproblems $C_{\mathrm{int}}$ and $C_{\mathrm{SIC}}$ and by modified waterfilling for subproblem $C_{\mathrm{SC}}$. It is given in Table 1, provided that the values of $\overline{P_{S_{\left.u_{[k}\right]}, n, \mathrm{SC}}}$ will be updated using the sequential Algorithm 2 [40]. This can be a general solution for the power optimization problem in an uplink underlay CR system, studied in [36] and [41]. The problem coefficients are presented in Table 2.

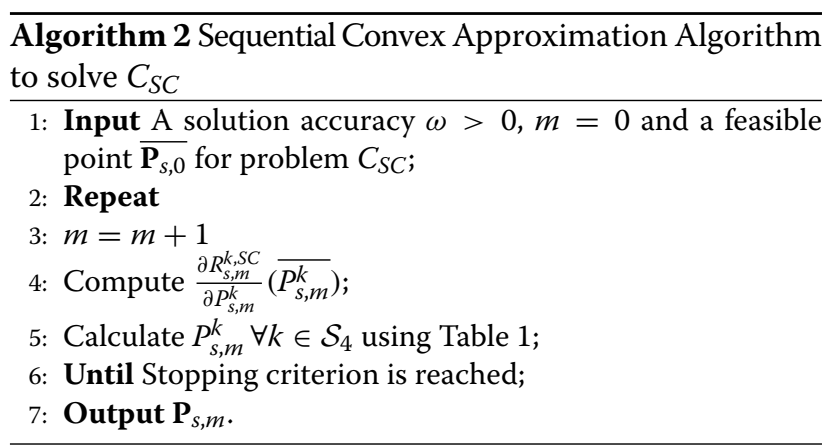

\section{Algorithm evaluation with imperfect CSI}

\subsection{System parameters}

In this section, it is assumed that the SUs have imperfect knowledge about the channel gains of the links between both primary and secondary transmitters and the primary receiver. This lack of knowledge results from channel estimation errors as well as feedback quantization errors. It is captured by adding a complex Gaussian noise to the channel gains $h_{p p}$ and $h_{p s}$. Consequently, the estimated channel gains are computed by:

$$
\begin{aligned}
\tilde{h}_{p p}^{k} & =h_{p p}^{k}+\gamma_{p p}, \forall k \\
\tilde{h}_{p s_{u_{[k]}}}^{k} & =h_{p s_{u_{[k]}}}^{k}+\gamma_{p s_{u_{[k]}}}, \forall k
\end{aligned}
$$

where $\gamma$ is a complex Gaussian noise. To simplify the notations, we assume that only one user is present in the secondary cell. We further assume that no outage is allowed at the SU.

\subsection{Algorithm}

In the first step, the estimated decoding strategy for any subcarrier $k$ is determined based on the estimated channel gains, i.e., $\tilde{h}_{p p}^{k}$ and $\tilde{h}_{p s}^{k}$, and is compared with the true decoding strategy determined by utilizing the exact values of channel gains, i.e., $h_{p p}^{k}$ and $h_{p s}^{k}$. If the estimated decoding strategy is more strict than the true one, outage on the $\mathrm{SU}$ is reported and the estimated achievable rate is set to zero. For example, if the SU makes a false judgment by

Table 1 Optimized values of $P_{s_{[k]}, n}^{k}$

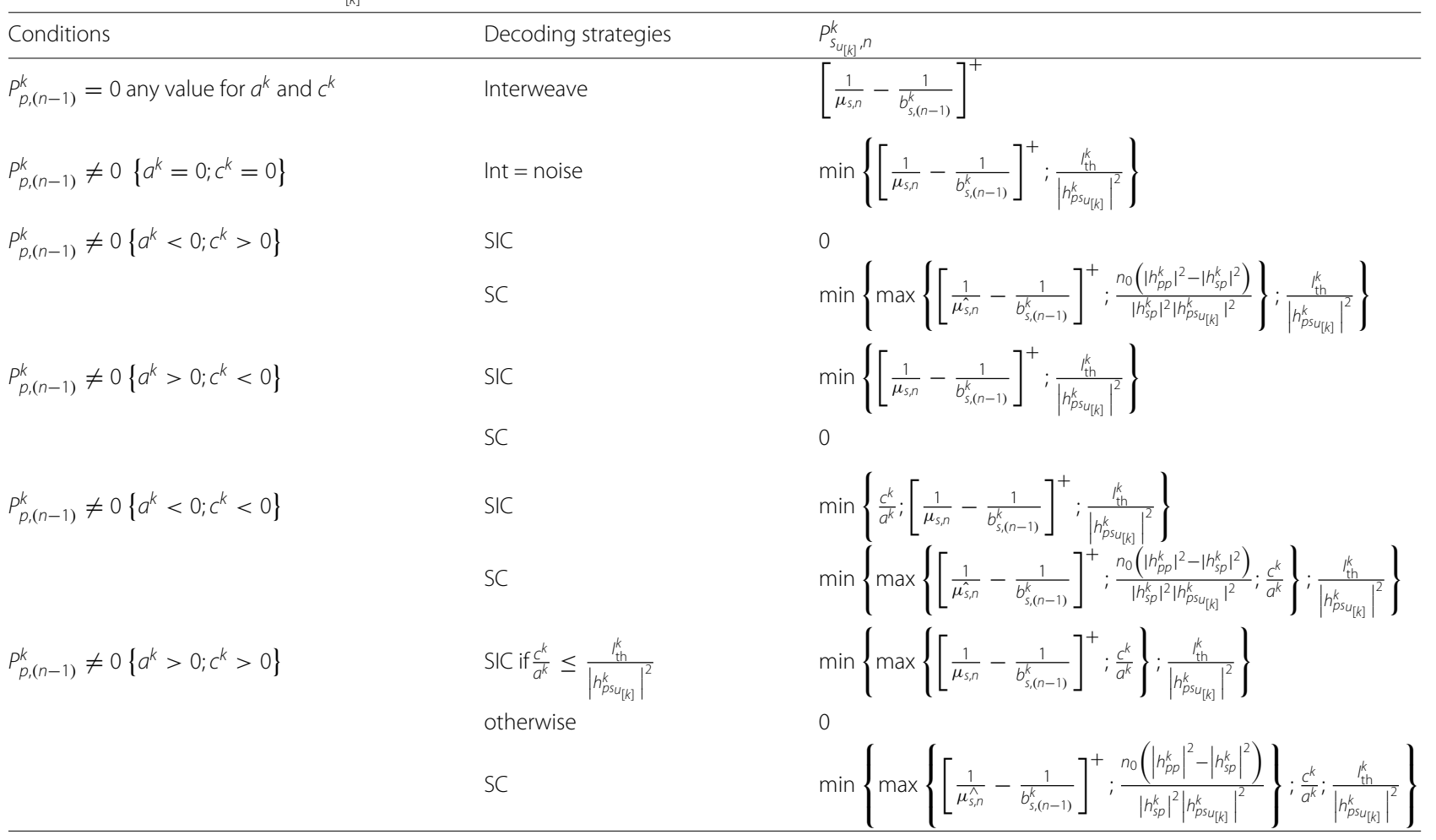


Table 2 Optimization coefficients for problem (16)

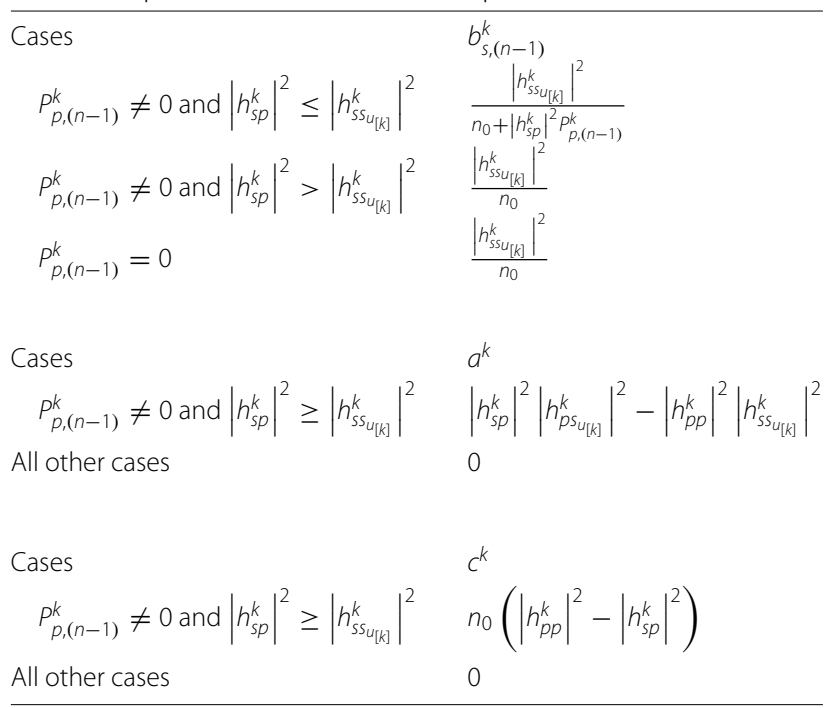

assuming it is able to apply SIC while in reality it is not because criterion (5) is not fulfilled with $h_{p p}^{k}$ and $h_{p s}^{k}$, then the secondary rate is set to zero.

Otherwise, if the estimated decoding strategy coincides with the true one, or if it is different than the true decoding strategy but still feasible at the SU, we proceed to the second step. This corresponds, for instance, to a case where the SU treats interference as noise although SIC could have been applied with the exact channel gains. Then, a test on the estimated achievable rate according to this estimated strategy is done. If this estimated rate is greater than the exact capacity calculated according to the true decoding strategy and the exact allocated power, the $\mathrm{SU}$ is in outage within the corresponding subcarrier and its rate is set to zero.

\section{Simulation results}

The performance of the proposed algorithm is assessed using Monte Carlo simulations, where the location of the primary and secondary transmitters both follow a uniform distribution. The performance is evaluated with perfect and imperfect CSI. In the former case, the performance of both single-user and multiuser cases is compared. The power constraint per transmitter is $21 \mathrm{dBm}$, and the thermal noise has a spectral density $N_{0}=-174 \mathrm{dBm} / \mathrm{Hz}$. The number of subcarriers is $L=64$ and $B=0.5 \mathrm{MHz}$ and $n_{0}=\frac{N_{0} B}{L}$. The allowed degradation on the primary rate due to interference from the secondary transmitter is $\epsilon=0.1$, which means that $90 \%$ of the interference-free rate is guaranteed. The influence of the distance between the primary and secondary BSs, $d_{\text {sec }}$ will be evaluated in the simulations. In all of our simulation results, all rates are normalized with respect to the bandwidth $B$. The complexity of the proposed algorithm is calculated as
$N(1+M) L \log _{2} L$, where $M$ is the number of iterations needed to update the Lagrange multiplier $\lambda$ in (14).

\subsection{Simulation results with perfect CSI}

With perfect CSI, the complex channel gains $h_{i j}^{k}$ take into account path loss, log-normal shadowing, and Rayleigh fading. We suppose here that all subcarriers are subject to independent Rayleigh fading. The path loss model is COST 231 extension to Hata model at $800 \mathrm{MHz}$ in dense urban environment, $L_{\mathrm{dB}}(d)=125.08+35.22 \times$ $\log _{10}(d)$, and the shadowing standard deviation is equal to $6 \mathrm{~dB}$. Both primary and secondary cells have omnidirectional antennas with the same radius $d_{p}=d_{s}=$ $1 \mathrm{~km}$. The stopping criterion of Algorithm 2 is either $\frac{\left|\sum_{k} R_{s, m}^{k, S C}\right|-\left|\sum_{k} R_{s, m-1}^{k, S C}\right|}{\left|\sum_{k} R_{s, m-1}^{k, S C}\right|}<\omega$ or $m=10$. It is important to note that for the power allocation algorithm, a convergence at $2 \%$ on the secondary rate is reached after six iterations.

\subsubsection{Performance evaluation for one secondary user \\ 5.1.2 Statistics}

In order to get an insight on the distribution of the different studied cases in terms of mobile terminal positions, we consider three different scenarios. In the first and second scenarios, the primary BS is considered as the center of reference for the terminal coordinates with $x_{\mathrm{BS}_{p}}=y_{\mathrm{BS}_{p}}=$ $0 \mathrm{~km}$, and the secondary BS is fixed at $(1 \mathrm{~km}, 0 \mathrm{~km})$.

In the first scenario, the position of the primary transmitter is fixed with $x_{p}=0.6 \mathrm{~km}$ and $y_{p}=0 \mathrm{~km}$ and the secondary transmitters are allowed to move along their own cell. The statistics of the decoding strategies given in terms of the percentage of applying each of them are represented in Fig. 3. We consider only two secondary users in the secondary cell. Once the user with the best data rate is activated within each subcarrier $k$, the statistics are executed.

The weak interference case, where the interference is treated as noise, depends exclusively on $\left|h_{s p}^{k}\right|^{2}$ and $\left|h_{s s_{\left.u_{[k]}\right]}}^{k}\right|^{2}$. With a large number of runs, represented by subfigure "Int," this case is only affected by the path loss and consequently by the distance between the selected SU and its BS. On the other hand, subfigures "SC" and "SIC" prove that these two methods are complementary and the application of SC in the algorithm will improve the system rates. When neither SIC nor SC can be applied, or whenever the interference constraints are not satisfied for all secondary users, the randomly chosen user is switched off. This case is inversely proportional to $\left|h_{p s_{u_{[k]}}}^{k}\right|^{2}$ as shown in subfigure "OFF".

In the second scenario, the position of the activated secondary transmitter is fixed at $x_{s}=1.4 \mathrm{~km}$ and $y_{s}=0 \mathrm{~km}$ and the primary transmitter is allowed to move along 


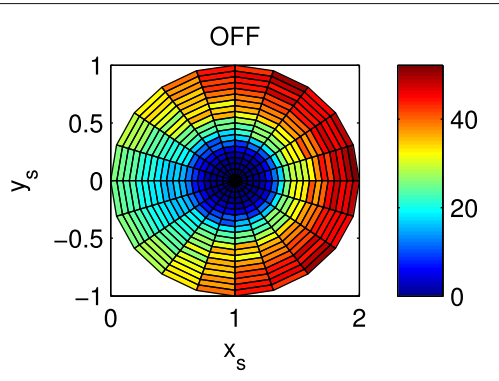

SIC
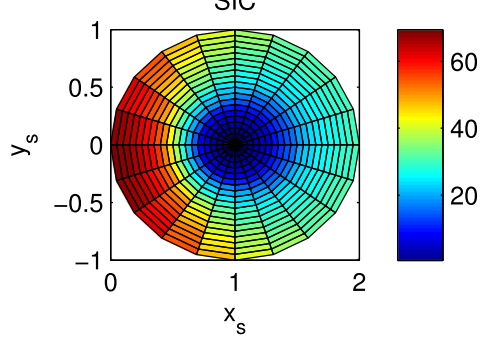

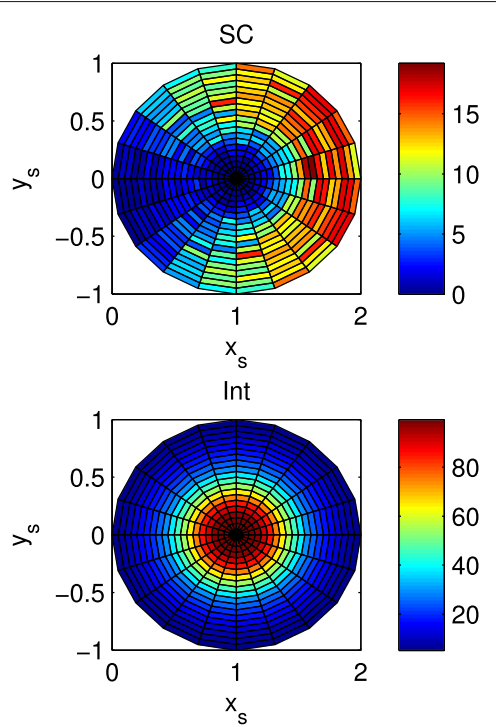

Fig. 3 Statistics of the decoding strategies for $x_{p}=0.6 \mathrm{~km}$ and $y_{p}=0 \mathrm{~km}$

its own cell. The statistics of all cases are represented in Fig. 4.

We can see that the interference is treated as noise when it is weak, i.e., whenever the distance between the PU and the secondary BS is greater than the distance between the secondary mobile and its BS, which is equal to $0.4 \mathrm{~km}$ in our case. Otherwise, the strong interference case is activated and several methods can be applied depending on the conditions studied previously. Since $\left|h_{s s_{u_{[k]}}}^{k}\right|^{2}$ is fixed, all cases depend on $\left|h_{s p}^{k}\right|^{2}$.

In the last scenario, the influence of both primary and secondary mobile terminal locations are evaluated. Both primary and secondary terminals are allowed to move along the $x$-axis. Both terminals are chosen to move between the two BSs. Statistics are given in terms of $d_{p}$ and $d_{s}$, the distance between each BS and its mobile (the activated secondary mobile within each subcarrier) (see Fig. 5).

When $d_{p}<d_{s}$, the interference is weak and is treated as noise at the secondary receiver. In the opposite side, SIC or SC can be used. The SU is turned off whenever either methods cannot be applied or the interference threshold is not satisfied.

\subsubsection{Rates improvement and comparison with several methods}

The impact of the proposed algorithm on the sum, primary, and secondary rates is shown in Figs. 6 and 7 .
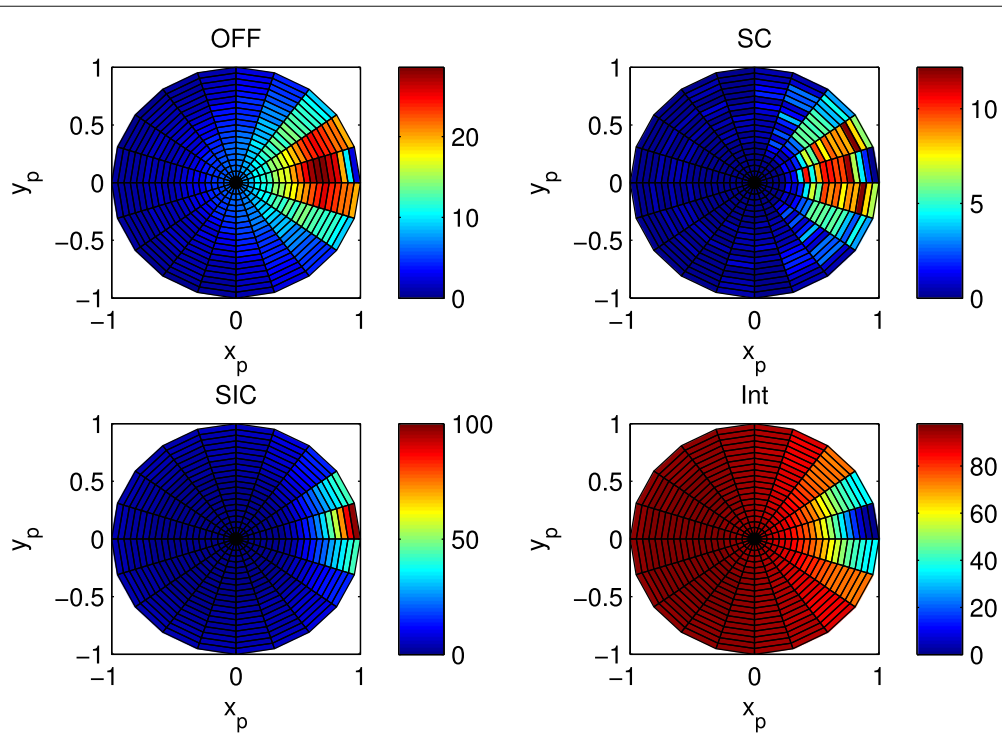

Fig. 4 Statistics of the decoding strategies for $x_{s}=1.4 \mathrm{~km}$ and $y_{s}=0 \mathrm{~km}$ 

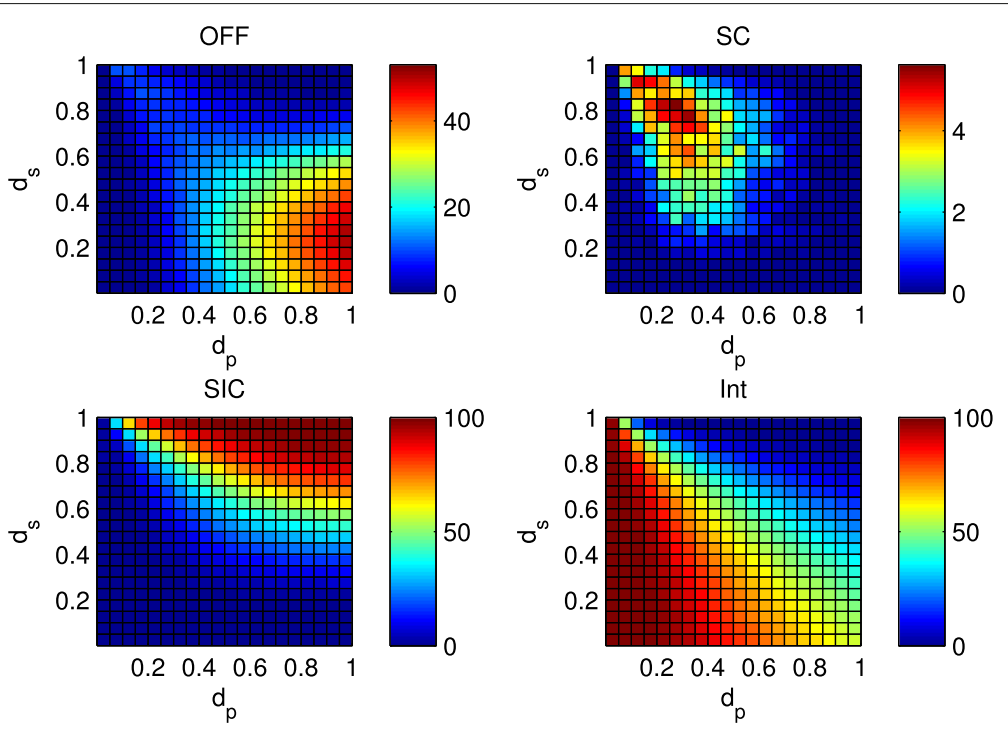

Fig. 5 Statistics of the decoding strategies for mobiles moving on $x$-axis between the BSs

By comparing the proposed method with the algorithm in [36] for the single-user scenario where only SIC is applied in the strong interference case, denoted by "SIC", the proposed method shows an improvement due to the application of SC. Applying only the superposition coding method (results referred to as "SIC \& SC"), an improvement that lies between 0.7 and $1.2 \%$ is obtained on the sum rate of the system, while the secondary rate increases between 5 and $17.3 \%$. On the other hand, the sum rate increases between 1.6 and $2.1 \%$ while the secondary rate increases between 11.5 and $25.4 \%$ when only power control is added to "SIC." These results are marked by "SIC \& power control" in the legend of both figures.

The improvement provided by the power optimization in presence of SC strategy is highlighted. When both superposition coding and dual decomposition are applied, the proposed algorithm denoted by "SIC \& SC \& power

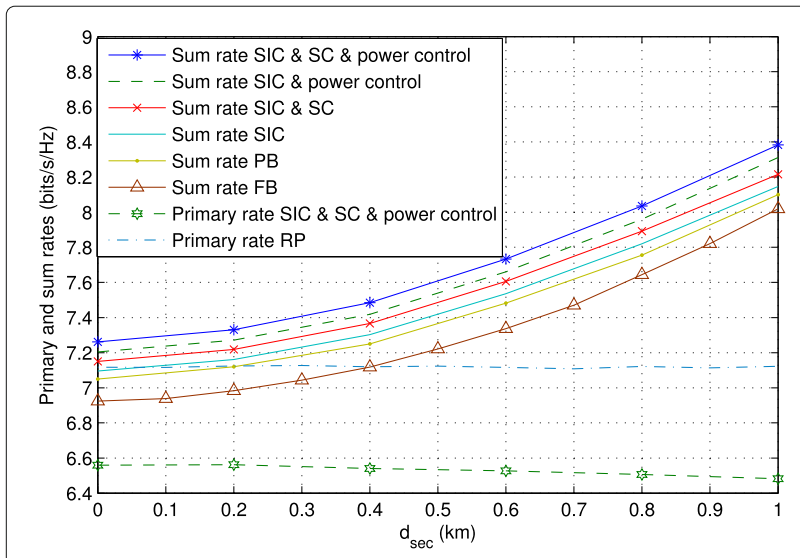

Fig. 6 Sum rate and primary rate compared with various methods control," the sum and secondary rates are compared with "SIC." The improvement is then between 2.3 and $3 \%$ on the sum rate, while it is between 16.9 and $43.5 \%$ on the secondary rate. In all these cases, there is a negligible degradation on the PU rate which never exceeds $0.7 \%$. This degradation is due to the increased power level allocated to the SU with our algorithm.

The proposed algorithm is also compared with three other schemes:

- The case where the secondary system is always switched off (denoted by "RP" for "Reference on Primary"). In this case, waterfilling is applied on the primary user and the complexity is calculated as $O\left(L \log _{2} L\right)$ [35].

- The classical power allocation scheme where the secondary system can transmit on the whole

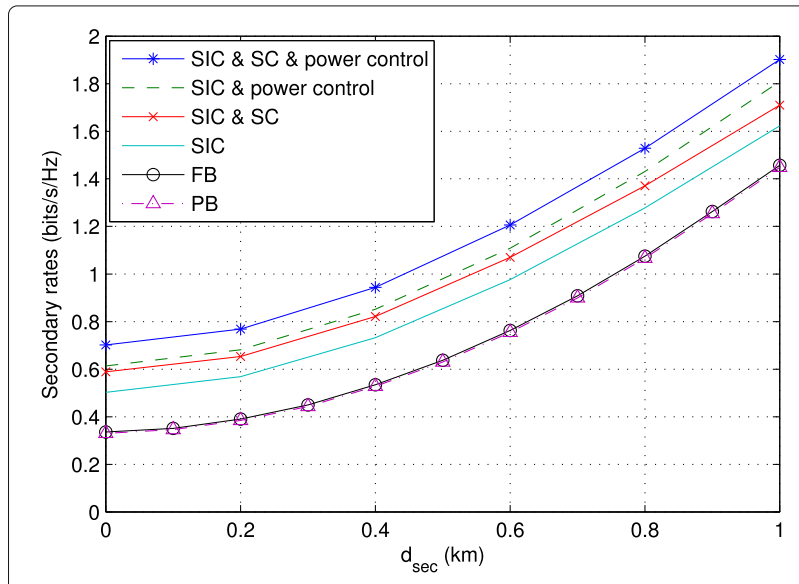

Fig. 7 Secondary rate compared with different methods 
bandwidth of a cognitive underlay/interweave system by considering the primary system's interference as noise in all subcarriers. This algorithm is denoted by "FB" for "Full Band" and its complexity is

$2 \cdot N \cdot O\left(L \log _{2} L\right)$, with $N$ as the number of iterations.

- An algorithm where the secondary system can only transmit in the subcarriers with weak interference $\left(\left|h_{s p}^{k}\right|^{2} \leq\left|h_{s s}^{k}\right|^{2}\right)$ by considering the primary system's interference as noise in these subcarriers. This algorithm is denoted by "PB" for "Partial Band," and its complexity is $3 / 2 \cdot N \cdot O\left(L \log _{2} L\right)$.

Both sum and primary rates are shown in Fig. 6. The adaptive decoding strategy with the proposed power control scheme achieves the highest sum rate. Compared with "RP," the achievable sum rate gain increases with $d_{\text {sec }}$ from 0.9 to $14.27 \%$. Moreover, the data rate gain obtained by comparing the proposed method with "FB" and "PB" is respectively $4.3-5.02 \%$ and $1.22-3.38 \%$.

The proposed method presents the lowest primary rate but the degradation comparing to " $\mathrm{FB}$ " does not exceed $0.8 \%$, where this gain reaches $4 \%$ when comparing with "PB," while the achieved gain on the secondary rate is about 40\%, as shown in Fig. 7.

To verify the convergence of the alternating optimization algorithm, we plot the evolution of the sum rate versus the number of iterations over a large number of channel realizations for $d_{\mathrm{sec}}=0.6 \mathrm{~km}$. Figure 8 confirms the convergence of the proposed algorithm despite the non-convexity of subproblem $C_{\mathrm{SC}}$ in (16c). This result is expected since the SCA monotonically increases the utility function and the objective function is bounded above due to power constraint and outage constraints.

\subsubsection{Performance evaluation with several secondary users} In this section, the performance of the proposed algorithm is evaluated with various numbers of mobile terminals in the secondary cell. In a first scenario, we suppose

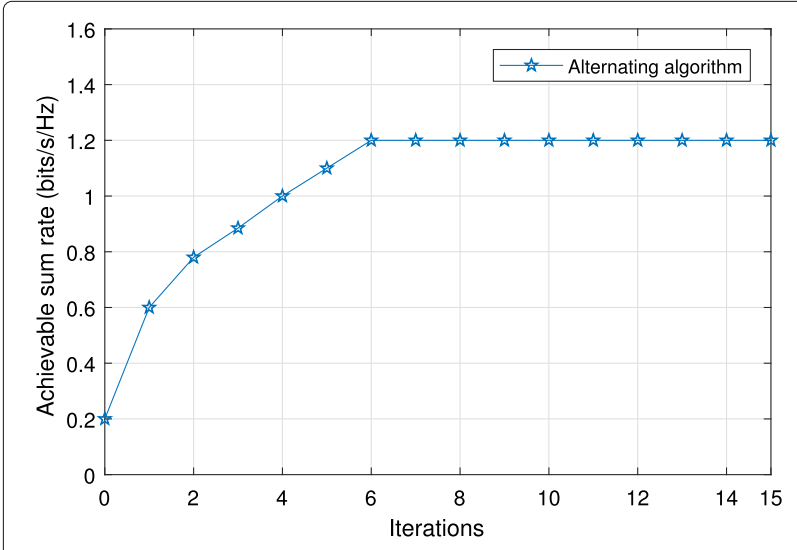

Fig. 8 Convergence of the proposed algorithm that only two SUs are present in the secondary cell. The influence of this multiuser diversity is presented in Fig. 9. The obtained gain for the sum rate, thanks to the multiuser aspect, has increased from $5.5-12 \%$ in a scenario which does not apply SC to $9.4-14 \%$ in our contribution.

The evolution of the secondary rates with respect to the number of mobiles in the secondary cell by considering the different scenarios (with and without SC, with and without power control) is drawn in Fig. 10, with $d_{\mathrm{sec}}=0.6 \mathrm{~km}$. The simulations show that the growth of the secondary rate increases from $9-14 \%$ when only SIC is applied to $21-31 \%$ when SIC, SC, and power control are applied.

Finally, the proposed algorithm is compared with exhaustive search, where the $\mathrm{SU}$ with the maximum estimated rate is allocated to each subcarrier, without selecting the users based on their decoding strategies. The achievable rates with the proposed algorithm are $4 \%$ less than those of the exhaustive search which represents an upper bound for the secondary rates. Consequently, we can conclude that our heuristic subcarrier allocation algorithm is less complex than the exhaustive search but still quite effective.

\subsection{Simulation results with imperfect CSI}

The performance of the proposed algorithm with imperfect CSI is assessed using Monte Carlo simulations, where the mobiles' positions follow a uniform distribution. We use the same parameters as in the former scenarios, replacing $h_{p p}^{k}$ and $h_{p s_{u_{[k]}}}^{k}$ by $\tilde{h}_{p p}^{k}$ and $\tilde{h}_{p s_{u_{[k]}}}^{k}$, respectively, where the variance of the complex Gaussian noise is equal to 0.01 . We reduce the maximum secondary power to $95 \%$, which decreases probability of being in outage at the secondary receiver. In this scenario, we do not allow either outage on rate or interference outage [42]. The impact of the proposed algorithm with imperfect CSI on both secondary and sum rates is shown in Figs. 11 and 12,

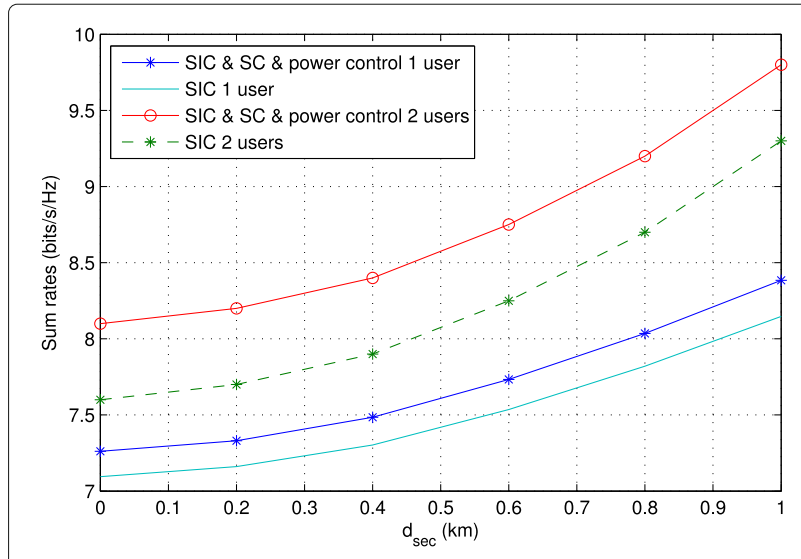

Fig. 9 Sum rates with two secondary users 


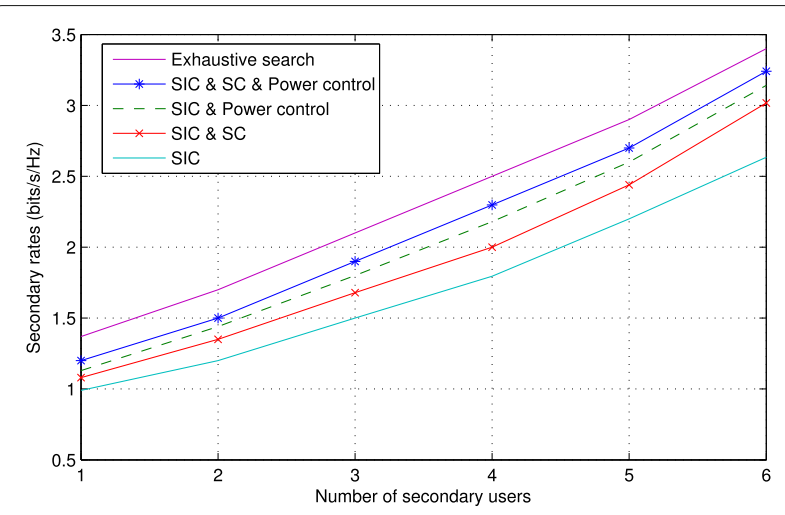

Fig. 10 Secondary rates depending on the number of users, for $d_{\text {sec }}=0.6 \mathrm{~km}$

respectively, for the single-user case. The estimated rates are denoted by "imperfect." Simulations show that the proposed algorithm with perfect CSI works well with noisy channels: there is always some gain due to the use of SC and the power allocation procedure. Moreover, the data rate gain obtained by comparing the proposed algorithm with "FB" is 10 and $4 \%$ on the secondary and sum rates, respectively, for small distance between the BSs.

\section{Conclusions}

In this paper, we proposed a resource allocation algorithm for a multiuser cognitive radio system. In a first step, an adaptive decoding algorithm based on both successive interference cancelation and superposition coding strategy is proposed in order to determine the achievable rates of different secondary users. After an orthogonal subcarrier allocation, the secondary rate maximization problem is formulated as a nonlinear problem and an iterative algorithm is proposed using waterfilling and dual decomposition methods. A general solution for the power

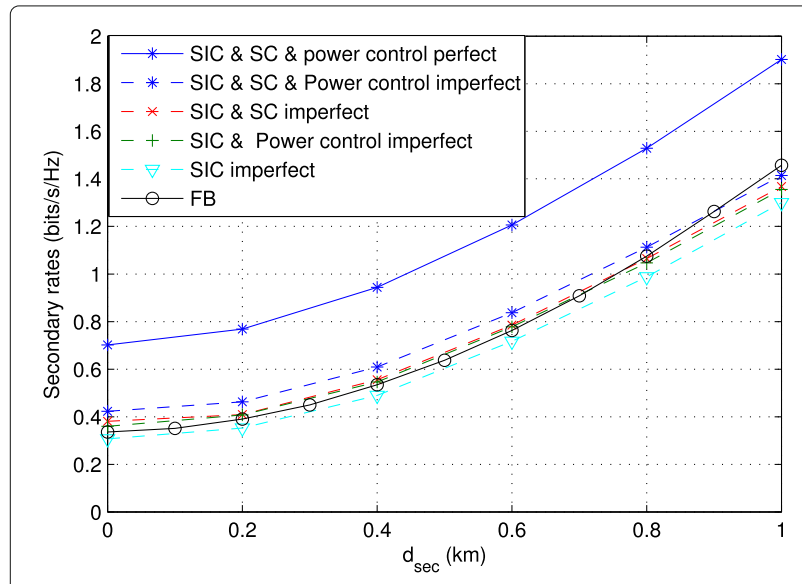

Fig. 11 Secondary rates with imperfect CSI

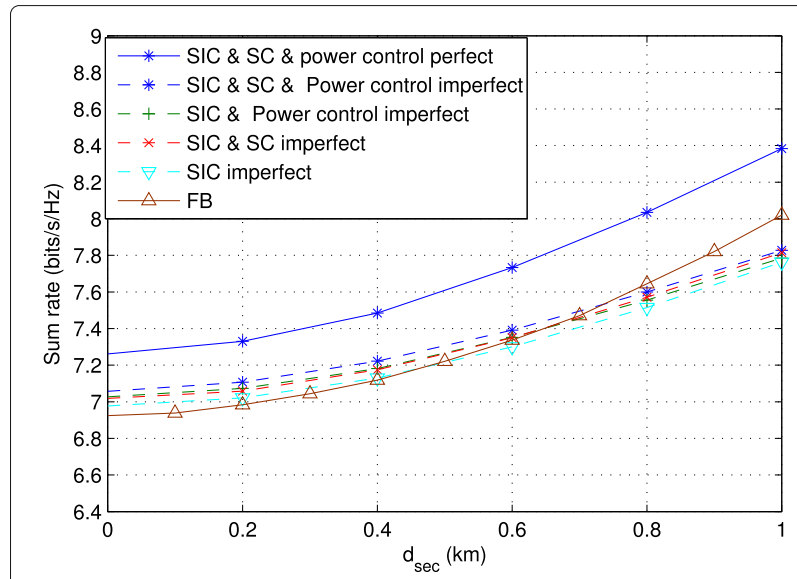

Fig. 12 Sum rates with imperfect CSI

optimization problem in an uplink underlay CR system is proposed. Both theoretical analysis and simulation results have shown that the proposed algorithm achieves higher sum rate than classical algorithms, providing high-enough data rates for the secondary system at the expense of a very low degradation of the primary system's rate. In the second part, we show by simulation analysis that the proposed algorithm is efficient and robust in the single-user case when imperfect CSI is available at the secondary user. For further work, the proposed algorithm could be extended to the statistical CSI case.

\section{Appendix 1}

Proof of Eq. (8)

Using SC, the secondary transmitter has then to transmit $x_{s}^{k}=\sqrt{\left(1-\alpha^{k}\right)} x_{s}^{k,(1)}+\sqrt{\alpha^{k}} x_{s}^{k,(2)}$, where $0 \leq \alpha^{k} \leq 1$, such that the secondary receiver receives $y_{s}^{k}=h_{s p}^{k} x_{p}^{k}+$ $h_{s s}^{k}\left(\sqrt{\left(1-\alpha^{k}\right)} x_{s}^{k,(1)}+\sqrt{\alpha^{k}} x_{s}^{k,(2)}\right)+z_{s}^{k}$.

- Step 1: $x_{s}^{k,(1)}$ is decoded from $y_{s}^{k}$ by treating $\sqrt{\alpha^{k}} h_{s s^{k}}^{k} x_{s}^{k,(2)}+h_{s p}^{k} x_{p}^{k}$ as noise, then $y^{k^{\prime}}=y^{k}-h_{s s}^{k} \sqrt{\left(1-\alpha^{k}\right)} x_{s}^{k,(1)}$ is obtained. The achievable rate is equal to:

$$
R_{s}^{k,(1)}=\frac{B}{L} \log _{2}\left(1+\frac{\left(1-\alpha^{k}\right)\left|h_{s s}^{k}\right|^{2} P_{s}^{k}}{\alpha^{k}\left|h_{s s}^{k}\right|^{2} P_{s}^{k}+\left|h_{s p}^{k}\right|^{2} P_{p}^{k}+n_{0}}\right)
$$

- Step 2: $x_{p}^{k}$ is decoded from $y^{k^{\prime}}$ by treating $\sqrt{\alpha^{k}} h_{s s}^{k} x_{s}^{k,(2)}$ as noise, then $y^{k^{\prime \prime}}=y^{k^{\prime}}-h_{s p}^{k} x_{p}^{k}$ is obtained. Thus, we have

$$
R_{p}^{k}=\frac{B}{L} \log _{2}\left(1+\frac{\left|h_{s p}^{k}\right|^{2} P_{p}^{k}}{\alpha^{k}\left|h_{s s}^{k}\right|^{2} P_{s}^{k}+n_{0}}\right)
$$


- Step 3: $x_{s}^{k,(2)}$ is decoded from $y^{k^{\prime \prime}}$. Consequently

$$
R_{s}^{k,(2)}=\frac{B}{L} \log _{2}\left(1+\frac{\alpha^{k}\left|h_{s s}^{k}\right|^{2} P_{s}^{k}}{n_{0}}\right)
$$

Consequently, the secondary achievable rate is calculated as

$$
\begin{aligned}
R_{s, n}^{k}= & \frac{B}{L} \log _{2}\left(1+\frac{\alpha^{k}\left|h_{s s}^{k}\right|^{2} P_{s, n}^{k}}{n_{0}}\right) \\
& +\frac{B}{L} \log _{2}\left(1+\frac{\left(1-\alpha^{k}\right)\left|h_{s s}^{k}\right|^{2} P_{s, n}^{k}}{\alpha^{k}\left|h_{s s}^{k}\right|^{2} P_{s, n}^{k}+\left|h_{s p}^{k}\right|^{2} P_{p, n}^{k}+n_{0}}\right)
\end{aligned}
$$

whence we got Eq. (8).

\section{Appendix 2}

\section{Proof of Eq. (9)}

The PU's rate seen by the primary's receiver $\left(R_{p, y_{p}}^{k}\right)$ is equal to the PU's rate seen by the secondary receiver $\left(R_{p, y_{s}}^{k}\right)$ :

$$
\frac{\left|h_{p p}^{k}\right|^{2} P_{p}^{k}}{\left|h_{p s}^{k}\right|^{2} P_{s}^{k}+n_{0}}=\frac{\left|h_{s p}^{k}\right|^{2} P_{p}^{k}}{\alpha^{k}\left|h_{s s}^{k}\right|^{2} P_{s}^{k}+n_{0}}
$$

From this equality, we get

$$
\alpha^{k}=\frac{\left(\left|h_{s p}^{k}\right|^{2}-\left|h_{p p}^{k}\right|^{2}\right) n_{0}+\left|h_{p s}^{k}\right|^{2}\left|h_{s p}^{k}\right|^{2} P_{s}^{k}}{\left|h_{p p}^{k}\right|^{2}\left|h_{s s}^{k}\right|^{2} P_{s}^{k}}
$$

which corresponds to Eq. (9) in the paper.

\section{Appendix 3}

\section{Proof of inequalities (7a) and (7b)}

$\alpha^{k}$ must be between 0 and 1 , so

$$
\begin{aligned}
0 & <\left(\left|h_{s p}^{k}\right|^{2}-\left|h_{p p}^{k}\right|^{2}\right) n_{0}+\left|h_{p s}^{k}\right|^{2}\left|h_{s p}^{k}\right|^{2} P_{s}^{k} \\
& <\left|h_{s s}^{k}\right|^{2}\left|h_{p p}^{k}\right|^{2} P_{s}^{k}
\end{aligned}
$$

which is equivalent to

$$
\begin{aligned}
\frac{\left(\left|h_{p p}^{k}\right|^{2}-\left|h_{s p}^{k}\right|^{2}\right)}{\left|h_{p s}^{k}\right|^{2}\left|h_{s p}^{k}\right|^{2}} & <\frac{P_{s}^{k}}{n_{0}} \\
& <\frac{\left|h_{p p}^{k}\right|^{2}\left|h_{s s}^{k}\right|^{2}}{\left|h_{p s}^{k}\right|^{2}\left|h_{s p}^{k}\right|^{2}} \frac{P_{s}^{k}}{n_{0}}+\frac{\left(\left|h_{p p}^{k}\right|^{2}-\left|h_{s p}^{k}\right|^{2}\right)}{\left|h_{p s}^{k}\right|^{2}\left|h_{s p}^{k}\right|^{2}}
\end{aligned}
$$

Two conditions are finally obtained:

$$
\left\{\begin{array}{l}
\frac{\left(\left|h_{p p}^{k}\right|^{2}-\left|h_{s p}^{k}\right|^{2}\right)}{\left.\left.h_{p s}^{k}\right|^{2} h_{s p}^{k}\right|^{2}}<\frac{P_{s}^{k}}{h_{0}} \\
\frac{P_{s}^{k}}{n_{0}}\left(\left|h_{p s}^{k}\right|^{2}\left|h_{s p}^{k}\right|^{2}-\left|h_{s s}^{k}\right|^{2}\left|h_{p p}^{k}\right|^{2}\right)<\left(\left|h_{p p}^{k}\right|^{2}-\left|h_{s p}^{k}\right|^{2}\right)
\end{array}\right.
$$

These conditions can be rewritten as:

$$
\left\{\begin{array}{l}
\frac{\left(\left|h_{p p}^{k}\right|^{2}-\left|h_{s p}^{k}\right|^{2}\right)}{\left.\left|h_{p s}^{k}\right|^{2} h_{s p}^{k}\right|^{2}}<\frac{P_{s}^{k}}{n_{0}} \\
P_{s}^{k} a^{k}<c^{k}
\end{array}\right.
$$

from where we got inequalities (7a) and (7b).

\section{Funding}

This work was partially funded through the French National Research Agency (ANR) project ACCENT5 with grant agreement code: ANR-14-CE28-0026-02. This work is supported by the national project ACCENT5 under the grant ANR14CE28002601.

\section{Authors' contributions}

All authors read and approved the final manuscript.

\section{Competing interests}

The authors declare that they have no competing interests.

\section{Publisher's Note}

Springer Nature remains neutral with regard to jurisdictional claims in published maps and institutional affiliations.

\section{Received: 9 October 2016 Accepted: 15 October 2017}

Published online: 06 November 2017

\section{References}

1. J Mitola, in IEEE International Workshop on, Mobile Multimedia Communications 1999, (MoMuC'99). Cognitive radio for flexible mobile multimedia communications (IEEE, San Diego, 1999), pp. 3-10

2. N Devroye, T Vahid, V Mai, Cognitive radio networks: highlights of information theoretic limits, models and design. IEEE Signal Proc. Mag. 52(6), 12-23 (2008)

3. N Devroye, P Mitran, $\mathrm{V}$ Tarokh, Achievable rates in cognitive radio channels. IEEE Trans. Inf. Theory. 52, 1813-1827 (2006)

4. A Goldsmith, SA Jafar, I Maric, S Srinivasa, Breaking spectrum gridlock with cognitive radios: an information theoretic perspective. Proc. IEEE. 97, 894-914 (2009)

5. R Blasco-Serrano, J Lv, R Thobaben, E Jorswieck, M Skoglund, Multi-antenna transmission for underlay and overlay cognitive radio with explicit message-learning phase. EURASIP J. Wirel. Commun. Netw. 2013(1), 195 (2013)

6. H Mahmoud, T Yucek, H Arslan, OFDM for cognitive radio: merits and challenges. IEEE Trans. Wirel. Commun. 16(2), 6-15 (2009)

7. S Sadr, A Anpalagan, KRaahemifar, Radio resource allocation algorithms for the downlink of multiuser OFDM communication systems. IEEE Commun. Surv. Tutor. 11(3), 92-106 (2009)

8. R Zhang, YC Liang, S Cui, Dynamic resource allocation in cognitive radio networks. IEEE Signal Proc. Mag. 27(3), 102-114 (2010)

9. G Bansal, MJ Hossain, VK Bhargava, Optimal and suboptimal power allocation schemes for OFDM-based cognitive radio systems. IEEE Trans. Wirel. Commun. 7(11), 4710-4718 (2008)

10. S Wang, F Huang, ZH Zhou, Fast power allocation algorithm for cognitive radio networks. IEEE Commun. Lett. 15(8), 845-847 (2011)

11. Y Zhang, $C$ Leung, Resource allocation in an OFDM-based cognitive radio system. IEEE Trans. Commun. 57(7), 1928-1931 (2009)

12. SM Almalfouh, GL Stuber, Interference-aware radio resource allocation in OFDMA-based cognitive radio networks. IEEE Trans. Veh. Technol. 60(4), 1699-1713 (2011)

13. X Kang, YC Liang, A Nallanathan, HK Garg, R Zhang, Optimal power allocation for fading channels in cognitive radio networks: ergodic capacity and outage capacity. IEEE Trans. Wirel. Commun. 8(2), 940-950 (2009)

14. X Kang, HK Garg, YC Liang, R Zhang, Optimal power allocation for OFDM-based cognitive radio with new primary transmission protection criteria. IEEE Trans. Wirel. Commun. 9(6), 2066-2075 (2010)

15. S Ekin, MM Abdallah, KA Qarage, E Serpedin, Random subcarrier allocation in OFDM-based cognitive networks. IEEE Trans. Signal Process. 60(9), 4758-4774 (2012)

16. MG Khoshkholgh, NM Yamchi, K Navaie, H Yanikomeroglu, VCM Leung, KG Shin, Radio resource allocation for OFDM-based dynamic spectrum sharing: duality gap and time averaging. IEEE J. Sel. Areas Commun. 33(5), 848-864 (2015)

17. A Jovicic, P Viswanath, Cognitive radio: an information-theoretic perspective. IEEE Trans. Inf. Theory. 55, 3945-3958 (2006)

18. M Pischella, D Le Ruyet, in International Workshop on, Signal Processing Advances in Wireless Communications (SPAWC 2013). Cooperative allocation for underlay cognitive radio systems (IEEE, Darmstadt, 2013), pp. 1-5 
19. M Chami, M Pischella, D Le Ruyet, in Proceedings of European Wireless 201420th European Wireless Conference. Adaptive decoding strategy with superposition coding for cognitive radio systems (VDE, Barcelona, 2014), pp. 1-6

20. T Cover, Broadcast channels. IEEE Trans. Inf. Theory. 18, 2-14 (1972)

21. M Chami, M Pischella, D Le Ruyet, in 11th International Symposium on Wireless Communications Systems (ISWCS) 2014. Optimal power control for cooperative underlay cognitive system (IEEE, Barcelona, 2014), pp. 558-562

22. R Zhang, YC Liang, Investigation on multiuser diversity in spectrum sharing based cognitive radio networks. IEEE Commun. Lett. 14(2), 133-135 (2010)

23. S Wang, F Huang, M Yuan, S Du, Resource allocation for multiuser cognitive OFDM networks with proportional rate constraints. Int. J. Commun. Syst. 25(2), 254-269 (2012)

24. JP Hong, W Choi, in 2010 IEEE International Symposium on Information Theory Proceedings (ISIT). Capacity scaling law by multiuser diversity in cognitive radio systems (IEEE, Austin, 2010), pp. 2088-2092

25. M Ge, S Wang, Fast optimal resource allocation is possible for multiuser OFDM-based cognitive radio networks with heterogeneous services. IEEE Trans. Wirel. Commun. 11(4), 1500-1509 (2012)

26. SWang, F Huang, C Wang, Adaptive proportional fairness resource allocation for OFDM-based cognitive radio networks. IEEE Trans. Wirel. Commun. 19(3), 273-284 (2013)

27. S Wang, ZH Zhou, M Ge, C Wang, Resource allocation for heterogeneous cognitive radio networks with imperfect spectrum sensing. IEEE J. Sel. Areas Commun. 31(3), 464-475 (2013)

28. YY He, S Dey, Throughput maximization in cognitive radio under peak interference constraints with limited feedback. IEEE Trans. Veh. Technol. 61(3), 1287-1305 (2012)

29. Z Rezki, MS Alouini, Ergodic capacity of cognitive radio under imperfect channel-state information. IEEE Trans. Veh. Technol. 61(5), 2108-2119 (2012)

30. A Shojaeifard, H Saki, MM Mahyari, M Shikh-Bahaei, in 2014 IEEE International Conference on Communications (ICC). Resource allocation and interference management for adaptive modulation and coding-based OFDMA cognitive radio networks (IEEE, Sydney, 2014), pp. 5908-5913

31. HA Suraweera, PJ Smith, M Shafi, Capacity limits and performance analysis of cognitive radio with imperfect channel knowledge. IEEE Trans. Veh. Technol. 59(4), 1811-1822 (2010)

32. THan, K Kobayashi, A new achievable rate region for the interference channel. IEEE Trans. Inf. Theory. 27(1), 49-60 (1981)

33. DP Bertsekas, Nonlinear programming. (Athena scientific Belmont, USA, 1999)

34. A Ghasemi, ES Sousa, Spectrum sensing in cognitive radio networks: requirements, challenges and design trade-offs. IEEE Commun. Mag. 46(4), 32-39 (2008)

35. DP Palomar, JR Fonollosa, Practical algorithms for a family of waterfilling solutions. IEEE Trans. Signal Process. 53(2), 686-695 (2005)

36. M Pischella, D Le Ruyet, Adaptive resource allocation and decoding strategy for underlay multi-carrier cooperative cognitive radio systems. Trans. Emerg. Telecommun. Technol. 24, 748-761 (2013)

37. G Scutari, D Palomar, S Barbarossa, in Information Theory and Applications Workshop, 2007. Asynchronous iterative water-filling for Gaussian frequency-selective interference channels (IEEE, Cannes, 2007)

38. WC Li, RY Chang, KY Wang, CY Chi, in 2015 IEEE International Conference on Acoustics, Speech and Signal Processing (ICASSP). Energy-efficient precoding matrix design for relay-aided multiuser downlink networks (IEEE, Brisbane, 2015), pp. 3098-3102

39. S Boyd, L Vandenberghe, Convex optimization. (Cambridge University Press, UK, 2004)

40. K Schittkowsk, C Zillober, Sequential convex programming methods. (Springer Berlin Heidelberg, Berlin, 1995)

41. B Maham, P Popovski, X Zhou, A Hjorungnes, Cognitive multiple access network with outage margin in the primary system. IEEE Trans. Wirel. Commun. 10(10), 3343-3353 (2011)

42. L Musavian, S Aissa, Fundamental capacity limits of cognitive radio in fading environments with imperfect channel information. IEEE Trans. Commun. 57(11), 3472-3480 (2009) 